\title{
Computer Assisted Orthopaedic Surgery (CAOS) in der Hüftendoprothetik
}

Der Begriff der ärztlichen Kunst hat bislang einen hohen Stellenwert. Dieses „Können“ verleiht dem Arzt die Möglichkeit, individuell auf den Patienten und dessen spezielle Situation einzugehen und seine Krankheit zu behandeln. Passend zur Jahrtausendwende mit dem Aufbruch in das neue Millenium scheint der Begriff der ärztlichen Kunst im chirurgischen Bereich seinen Wert zu verlieren. Speziell in der Hüftendoprothetik und der Wirbelsäulenchirurgie heißt es, daß der Computer oder Roboter operationstechnisch besser sei als der ,(kunst-)handwerklich“ agierende Operateur $[3,4,8]$. Die Patienten sind bereits umfassend über die Vorteile der revolutionierenden Systeme informiert. Die Industrie reagiert sofort auf die verstärkte Nachfrage, ,denn die Einführung der computerassistierten Chirurgie im Operationssaal, bei der ein Roboter das Ausfräsen des Prothesenlagers im Femur übernimmt, zwingt die Mediziner und Implantatehersteller zur Entwicklung neuer Verankerungsmechanismen ..." (Auszug eines Firmenprospektes). Der Marktdruck steigt. Ganze Klinikketten haben sich Operationsroboter, vor allem für die Hüftchirurgie angeschafft.

Firmen und Kliniken verweisen gemeinsam auf die vorliegende Literatur, wonach nur Computer und Roboter mit ihrer Exaktheit bessere Operationsresultate erbringen können. Börner geht davon aus, daß ,aufgrund eines wesentlich verbesserten Knochenkontaktes, bedingt durch die hohe Präzision beim Fräsvorgang und der dadurch erreichbaren hohen Primärstabilität ein besseres Anwachsverhalten durch den Knochen gegeben sein wird“. So beträgt die Kontaktfläche zwischen Prothese und Knochenlager beim Fräsen durch den Roboter 95\%, bei Präparation des Markraumes mit der Raspel aber nur durchschnittlich 20\% (Paul et al. 1992). Bargar et al. aus derselben Arbeitsgruppe stellen allerdings die Frage, ob diese Technik wirklich einen Einfluß auf die langfristigen Ergebnisse hat. Obwohl die meisten Operateure einen $\mathrm{Zu}$ sammenhang zwischen der Exaktheit der Implantationstechnik und langfristigen Ergebnisse herstellen, gäbe es nämlich in der orthopädischen Literatur keine einzige Studie, die diesen Zusammenhang beweisen kann (1998).

Zurecht wird diese neue Technologie daher hinsichtlich ihrer Wertigkeit überprüft. In den USA liegt die Federführung bei der Food and Drug Administration, bei der jedoch eine gute Portion Skepsis bezüglich der Exaktheit, der Praktikabilität und vor allem der Kosteneffizienz vorherrscht (Breisch 1998). In der Bundesrepublik dagegen sind die Ergebnisse so präsentiert, daß der Laie - allgemeingebildet, zukunftsorientiert und technologiefreundlich - in Massen in die ,modernen“ Kliniken strömt. Chefärzte, ärztliche und Verwaltungsdirektoren beobachten die Marktentwicklung aufmerksam, zum Teil mit Sorge. Manche reagieren prompt und schaffen sich das vermeintlich bessere System rasch an, um wettbewerbsfähig zu bleiben. Wurde nicht jahrelang vorgerechnet, da $B$ in der Leistungsorientierung und im Wettbewerb die Zukunft eine kosteneffiziente Medizin liege? In den um die Wissen-

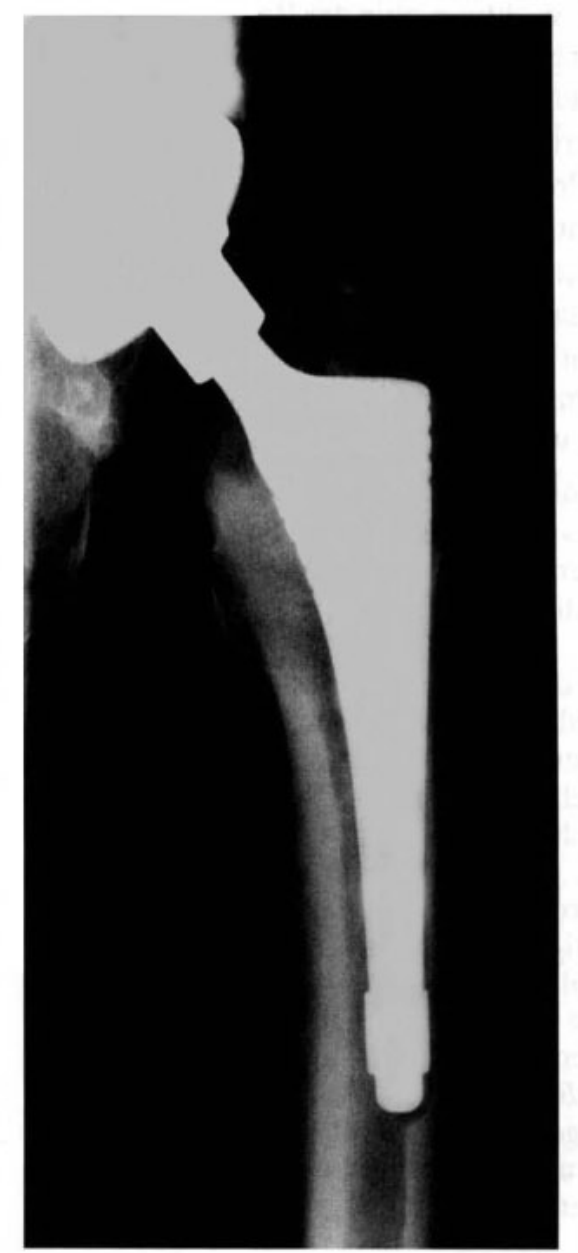

schaft bemühten Universitätsklinika ist dieser Prozeß gerade erst angelaufen.

Vielleicht liegt es daran, daß den Herausgebern der Zeitschrift für Orthopädie zum Thema der computerassistierten Hüftendoprothetik bisher keine Arbeiten vorgelegt wurden oder vorgelegt werden konnten. In weiteren Kreisen der universitären Orthopädie ist man wie bei der Food and Drug Administration dieser Technik gegenüber skeptisch eingestellt, ohne dies allerdings mit Zahlen oder Beobachtungen begründen zu können. Aus der Wirbelsäulenchirurgie ist bekannt, daß die computerassistierte Insertion von Pedikelschrauben exakter ist als die durch den orthopädischen Chirurgen vorgenommene (Laine et al. 1997, Schwarzenbach et al. 1997). Dessen ungeachtet ist die Exaktheit des Verfahrens bei weitem nicht $100 \%$. Laine et al. stellten fest, daß auch bei computerassistierter Insertion der Pedikelschrauben in 4,3\% eine Fehllage zu beobachten war. Schwarzenbach et al. zeigten, daß bei 2,7\% der Insertionen die Corticalis perforiert wurde.

Derartige Fehlinsertionen sind offenbar auch bei der computerassistierten Hüftendoprothetik möglich (Abb. 1). Er erstaunt, daß hierzu in der Literatur bisher nichts zu erfahren ist. Die Betrachtung dieses Bildes macht nachdenklich. Vielleicht erschüttert es sogar den Glauben an die Unfehlbarkeit der Roboterchirurgie in diesem Bereich. Zahlreiche Fragen werden aufgeworfen: 
1. Ist dies eine Fehlimplantation? - Die Kontaktfläche zwischen Prothese und Knochenmark mag wie in der Literatur behauptet auch in diesem Fall bei $95 \%$ liegen. Aber ist es nicht wichtig, wohin der Roboter fräst? Ist es denn prinzipiell günstig, wenn der Fräsvorgang die Corticalis tangiert und damit die Durchblutung und damit das Anwachsverhalten des Knochens an der zementfrei implantierten Prothese beeinträchtigt - Untersuchungen weisen darauf hin, daß die Biologie des Prothesenlagers für die Langzeitresultate von wesentlicher $\mathrm{Be}$ deutung ist (Draenert et al. 1999).

Wenn bei einem jungen Menschen mit kräftigem Knochen mit herkömmlicher Implantationstechnik die Corticalis angefräst wird, wie dies in Abb. 1 zu erkennen ist, kann das allenfalls mit Ungestüm und Unerfahrenheit des Operatueurs begründet werden. Ist die Abb. 1 etwa so zu interpretieren, da $B$ der Operationsroboter zumindest augenblicklich noch „ungestüm und unerfahren ” ist?

2. Wie konnte das dem Chirurgen passieren? War er etwa schlecht ausgebildet oder war er unkonzentriert? Liegt der Fehler an der bisher nicht erforschten Schnittstelle zwischen Mensch und Maschine (Visarius et al. 1997)?

3. Wo liegt der Fehler und wer ist verantwortlich? Ist es der Operateur, die Herstellerfirma des Operationsroboters, der Programmierer oder der Radiologe, der das CT angefertigt hat? Oder sind es etwa alle?

4. Wird dieser Fehler wieder eintreten? Wie ist er zu vermeiden? Ist eine genaue Ursachenforschung möglich?

5. Ist das Verfahren überhaupt kosteneffizient? - Immerhin werden von Bargar et al. $3,2 \%$ TEP-Luxationen und $3,9 \%$ (vorübergehende) Nerveläsionen beschrieben. Operationszeit und Blutverlust sind bei den roboteroperierten Patienten signifikant größer.

„Die Zukunft ist da - aber funktioniert sie auch?“ Diese Frage wurde von Breisch im AOOS-Bulletin zurecht gestellt. Die computerassistierte orthopädische Chirurgie befindet sich im Entwicklungs- und Versuchsstadium. Sie muß für jede Indikation eingehend evaluiert werden. Ohne Zweifel kann die Computerunterstützung die Exaktheit von bestimmten Operationsabläufen verbessern. Für den allgemeinen Einsatz der computerassistierten orthopädischen Chirurgie gilt allerdings, daß damit die Kunst des Operateurs nicht ohne weiteres ersetzt werden kann. Stößt der Operateur bei der Insertion einer Predikelschraube auf Schwierigkeiten, so wird er diese im Zweifelsfall immer nach lateral orientieren, weil der Gefahrenherd medial, nämlich im Spinalkanal lauert. Der Computer ist zumindest heute noch "dumm" und kann diesen Zweifelsfall bisher nicht erkennen (Schwarzenbach et al. 1997). Dieses Feeling des Operateurs wird auch beim Erfahrenen dazu führen, daß dieser bei herkömmlicher Implantation kaum in die Gefahr geraten dürfte mit der Raspel einen Femurschaft zu perforieren oder die Corticalis zu lädieren, wie dies in Abb. 1 zu erkennen ist.

Für die computerunterstützte Hüftendoprothetik gilt speziell, daß die Ergebnisse der zementierten und nichtzementierten Endoprothetik derzeit bereits erfolgversprechend sind und langfristig gute Resultate aufweisen (Malchau et al. 1993). Ob die computerassistierte Hüftendoprothetik überhaupt einen Fortschritt zu erbringen vermag, wird sich wahrscheinlich erst in 10-15 Jahren zeigen.
Wie wird sich der Markt weiterentwickeln? Zwei Möglichkeiten bieten sich an:

1. Alle kaufen sich einen Operationsroboter, bis der Markt gesättigt ist. Alle machen ihre Erfahrungen, bis sich die Spreu vom Weizen getrennt hat und in 10 bis 15 Jahren über die langfristigen Erfolge berichtet werden kann. Dieser Weg ist teuer.

2. Analog der in den USA von der Food and Drug Administration ausgesprochenen beschränkten Zulassung zum klinischen Versuch werden nur verschiedene Kliniken für die Evaluation ausgesucht. In diesem Fall müßten sich die wissenschaftlichen Gesellschaften der an diesem Verfahren interessierten Berufsgruppen für dieses Verfahren einsetzen und sich die Kliniken selbst beschränken. Bereits getätigte Investionen zahlreicher Kliniken wären nutzlos. Dieser Weg ist wenig realistisch.

Wir werden uns also mit dem Weg der Marktsättigung anfreunden müssen. Es ist zu hoffen, daß die computerassistierte orthopädische Chirurgie (CAOS) dabei den ihr gebührenden Stellenwert erhält und nicht durch überschießende Entwicklung im Chaos endet.

\section{F. U. Niethard, Aachen}

\section{Literatur}

${ }^{1}$ Bargar, L., E. J. Carbone: Robotic femoral canal preparation. In: Total hip revision (Galante, J. O., A. G. Rosenberg, J. J. Callaghan, Eds.), Raven Press, L.T.D. New York 1995

${ }^{2}$ Bargar, W. L., A. Bauer, M. Börner: Primary and revision total hip replacement using the robodoc system. Clinical orthpaedics related research 354 (1998) 82-91

${ }^{3}$ Börner, M., A. Bauer, A. Lahmer: Unfallchirurg 100 (1997) 640-645

${ }^{4}$ Börner, M., A. Bauer, A. Lahmer: Rechnerunterstützter Robotereinsatz in der Hüftendoprothetik. Orthopädie 26 (1997) 251-257

${ }^{5}$ Breisch, S. L.: Future is here - does it work? AAOS Bulletin (1998) 35-36

${ }^{6}$ Di Gioia, A. M., B. Jaramaz, B. D. Colgan: Computer assistet orthopaedic Surgery Clinical orthopaedics related research 354 (1998) 8-16

7 Draenert, K., Y. Draenert, U. Garde, Ch. Ulrich: Manual of Cementing Technique. Springer, Heidelberg, New York 1999

${ }^{8}$ Laine, T., D. Schlenzka, K. Makitalo, K. Tallroth, L. P. Nolte, H. Wisarius: Improved acurisy of pedicel stru insertion with computer assistet Surgery. A prospectiv clinical trial of 30 patients. Spine 22 (1997) 1254-1258

${ }^{9}$ Malchan, H., P. Herbert, L. Ahnefeldt, O. Johnell: Prognose der totalen Hüftarthroplastik. 61. Annual Meeting der American Academy of Orthopaedic Surgeons, San Francisco 1993

${ }^{10}$ Paul, H. A., W. L. Bargar, B. Mittelstadt, B. Musits, R. H. Taylor; P. Kazanzides, J. Zuhars, B. Williamson, W. Hanson: Development of a surgical robot for cementless total hip arthroplasty. Clin. Orthop. rel. Research 285 (1992) 57-66

$"$ "Schwarzenbach, O., U. Berlemann, B. Jost, H. Wisarius, E. Arm, F. Langlotz, L. P. Nolte, C. Ozdoba: Acurisy of computer assistet pedicel scrue placement an in vivo computed tomography analysis

12 Simon, D. A., S. Lavallée: Medical imaging and registration in computer assistet Surgery Clinical orthopaedics related research 354 (1998) 17-27

${ }^{13}$ Visarius, H., J. Gong, C. Scheer, S. Haralamb, L. P. Nolte: Men machine interfaces in computerassistet Surgery. Comput aides surgery 2 (1997) 102-107 